\title{
LOS GRUPOS DE AUTOAYUDA COMO ESTRATEGIA DE INTERVENCIÓN EN EL APOYO SOCIAL
}

\author{
YOLANDA DOMENECH LÓPEZ
}

Profesora titular de Trabajo Social. E.U.T.S. Universidad de Alicante.

\section{RESUMEN}

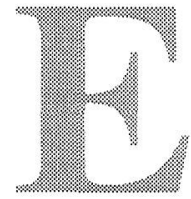

1 presente artículo pretende explicar qué son los grupos de autoayuda y cómo podemos, desde el trabajo social, crear y ayudar a que estos grupos se desarrollen.

En primer lugar, se analizan algunos aspectos teóricos del Apoyo social como teoría clave en los grupos de autoayuda (es decir, en un primer momento se trata de decir qué justifica la creación de grupos de autoayuda, entendidos estos como una de las estrategias fundamentales para conseguir apoyo). En segundo lugar, centrándonos en la falta de apoyo y en la necesidad de estructurar la red social de algunas personas, se explican los fundamentos teóricoprácticos de los grupos de autoayuda, como una forma especial de dar apoyo: darlo en grupo y con el grupo.

\section{PALABRAS CLAVE}

Apoyo social, grupos de autoayuda, red social

\section{CONSIDERACIONES PREVIAS: ASPECTOS TEÓRICOS DEL APOYO SOCIAL.}

El concepto de apoyo social surge alrededor de los años setenta cuando los resultados de diversas investigaciones acerca de problemáticas sociales comienzan a demostrar que la falta de apoyo es un elemento común a todas ellas. Esta falta de apoyo se hace evidente en la carencia de interacciones o en la ruptura de relaciones (como más tarde se ha venido llamando en la ausencia o ruptura de redes sociales).

No obstante, aunque en la práctica se operativiza, no es fácil definir qué es el apoyo social, ya que son numerosas las actividades que se incluyen bajo este término. Normalmente, se utiliza el concepto de red 
social para referirse a él, aunque, como se explica a continuación, son aspectos diferenciados ya que la red social podría definirse como la «socioestructura» donde tienen lugar las transacciones de apoyo.

Tres son las líneas que definen el apoyo social, haciendo hincapié en un aspecto u otro según nos situemos. A modo de síntesis, destacar algunas de las características de cada línea y explicar cómo, a través del grupo, puede operativizarse el apoyo social que, desde cada perspectiva, se plantea.

\subsection{Perspectivas del apoyo social}

1. La perspectiva estructural enfatiza la importancia de las características de las redes sociales y de las condiciones objetivas que rodean el proceso de ayuda. El interés se centra en los elementos más identificables y objetivos de la red de relaciones de una persona (Gracia, 1997: 23). Desde esta perspectiva se define el apoyo social en términos del número de relaciones que mantiene el sujeto, la interconexión de las personas con las que se relaciona, el equilibrio o desequilibrio de sus intercambios y la semejanza o congruencia entre los miembros de la red.

La creación de grupos de autoayuda tiene sentido en el momento que se detecta que una persona a penas posee red social, no existe homogeneidad (por ejemplo, una joven madre soltera donde toda su red son personas de mayor edad), o los intercambios no se dan de forma recíproca, pudiendo aparecer conductas sobreprotectoras, excesivo control, abandonos, etc. Estos grupos ayudan a crear y/o ampliar una parte de red social que proporcione el apoyo necesario a las personas que forman parte del grupo.

2. La perspectiva funcional se centra en las funciones que cumplen las relaciones sociales. Desde esta perspectiva, Caplan (1974) define el apoyo social como guía y feedback proporcionado por otras personas que permite al sujeto manejar emocionalmente un evento estresante. Existe un acuerdo entre los investigadores en distinguir tres funciones fundamentales en el apoyo social, funciones que se dan en los grupos de autoayuda:

a) Función de apoyo emocional, a través de la cual las personas comparten experiencias y se sienten aceptadas por los demás.

b) Función de apoyo instrumental o material donde se intercambian ayudas que sirvan para resolver problemas prácticos y cotidia-

I En definitiva, el apoyo social se centra en el estudio de los cuatro elementos más importantes de la red y su relación con el sujeto. Estos son el tamaño, la densidad, la reciprocidad y la homogeneidad de la red. 
nos. En los grupos de autoayuda esta función suele darse en las fases avanzadas del grupo, ya que en las primeras fases las personas suelen ser reacias a pedir este tipo de ayuda. Además, suele darse fuera del contexto grupal.

c) Función de apoyo informacional, donde a través del proceso grupal las personas reciben información y guía.

Por tanto, desde la perspectiva funcional, el apoyo social se define como las transacciones en las que se intercambian recursos que permiten cumplir alguna de estas tres funciones (Barrón, 1996:17). A través de los grupos de autoayuda se ofrecen e intercambian diversos recursos, que permiten cumplir estas tres funciones.

3. La perspectiva contextual, enfatiza las características contextuales del apoyo social. Desde aquí, se debe adecuar el tipo de apoyo al tipo de problema en el que se trabaja. A veces, la propia familia no puede dar apoyo en una situación determinada y debemos recurrir a otras fuentes de apoyo como pueden ser los amigos o los grupos. Esta línea, la contextual, también considera importante la duración del apoyo en cada situación. Los grupos de ayuda, por ejemplo, ofrecen apoyo a largo plazo para aquellas personas que padecen situaciones crónicas.

Estas tres perspectivas ayudan a definir el concepto de apoyo social resaltando diferentes aspectos según nos situemos en una o otra. No obstante, el apoyo social puede operativizarse de múltiples formas, tal y como se señala en el cuadro $n^{\circ} 1$ que se presenta. Las intervenciones basadas en el apoyo social, independientemente de la estrategia que se adopte (en nuestro caso el grupo de autoayuda), implican la interacción

\section{CUADRO N. ${ }^{\circ}$}

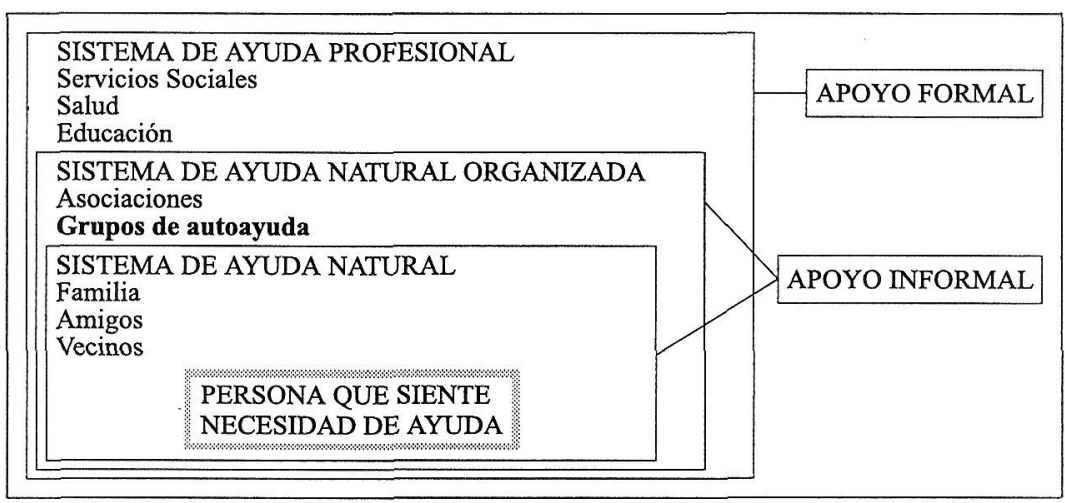

FUENTE: Adaptación de Villalba Quesada, C.: «Redes sociales: Un concepto con importantes implicaciones en la intervención comunitaria». Intervención Psicosocial, 4. 1993. Pág. 69-85. 
directa con el entorno social de la persona y sus efectos positivos son resultado de los recursos que se intercambian durante esa interacción (Gracia, 1997:39-40).

\section{APLICACIONES PRÁCTICAS: LOS GRUPOS DE AUTOAYUDA COMO ESTRATEGIA DE INTERVENCIÓN.}

Los grupos de autoayuda surgen como alternativa al sistema tradicional donde la dirección y la responsabilidad corre a cargo de un profesional. Este movimiento, el de la autoayuda, creció y se desarrolló de forma paralela al desarrollo de la profesión, a partir de los años 30, con la creación de Alcohólicos Anónimos.

Tal y como veíamos en la primera parte, estos grupos suponen para el Trabajo Social una estrategia de intervención centrada en el apoyo social. Teniendo como objetivo consolidar, ampliar y complementar la red social del individuo que atraviesa alguna dificultad y/o problema no pudiendo recibir apoyo o no siendo adecuado el apoyo que recibe de otros sistemas de su red social. Estos grupos han surgido en campos tan diversos como la enfermedad mental y física, las adicciones, las crisis vitales, y diversas problemáticas sociales.

No obstante, abogar en defensa de la creación y el trabajo a través de estos grupos no supone olvidar todo el trabajo complementario que debemos hacer si queremos trabajar desde un enfoque que englobe al individuo y al conjunto de sus sistemas.

\subsection{Concepto}

Entre las numerosas definiciones que se han propuesto, destacamos a continuación la definición propuesta por Katz y Bender (1976), definición que ha adoptado la Organización Mundial de la Salud. Esta dice así: «los grupos de autoayuda son grupos pequeños y voluntarios estructurados para la ayuda mutua y la consecución de un propósito específico. Estos grupos están integrados habitualmente por iguales que se reúnen para ayudarse mutuamente en la satisfacción de una necesidad común, para superar un handicap común o problemas que trastornan la vida cotidiana, y conseguir cambios sociales y/o personales deseados. Los iniciadores y miembros de estos grupos perciben que sus necesidades no son o no pueden ser satisfechas por las instituciones sociales existentes. Los grupos de ayuda mutua enfatizan la interacción social cara a cara y la responsabilidad personal de sus miembros. Con frecuencia, proporcionan ayuda material así como apoyo emocional; están orientados a la causa del problema y promueven una 
ideología o conjunto de valores a través de los cuales los miembros del grupo pueden obtener e incrementar un sentimiento de identidad personal».

\subsection{Características}

Son numerosos los autores que identifican las características de los grupos de autoayuda (Killilea, 1976; Richardson,1983; Rogers, 1987; Orford,1992 ...). Siguiendo a Gracia (1997), destacamos, como características más relevantes de los grupos de autoayuda, las siguientes:

\subsubsection{Los miembros del grupo intercambian experiencias comunes}

El hecho de compartir un problema central define el estatus de miembro en los grupos de autoayuda, a pesar de las numerosas diferencias individuales. En este sentido, Silverman (1970) también considera que entre las principales características de los grupos de autoayuda se encuentra el hecho de que quien provee ayuda comparte el mismo problema con quien recibe esa ayuda. Para este autor, la experiencia común es el concepto fundamental que distingue la experiencia de la autoayuda de otros intercambios de ayuda.

Además, en estos grupos, la ayuda mutua no sólo surge de la experiencia común de un mismo problema sino de los sentimientos similares acerca de ese problema. Y de las experiencias comunes de resolución de conflictos aparecidos como consecuencia del malestar generado.

\subsubsection{El grupo proporciona apoyo emocional.}

Por apoyo emocional se entiende el hecho de saber acompañar y entender a otro miembro del grupo que padece un problema. El apoyo emocional que se transmite puede ayudar a la persona bien a ajustarse a la situación o bien a cambiar esa situación (Richardson,1883). Este apoyo se da durante las reuniones del grupo, pero a veces también puede darse fuera del grupo.

En el contexto de los grupos de autoayuda el apoyo emocional puede adoptar diversas formas. Entre otras conductas, los miembros del grupo proveen apoyo emocional cuando (Katz, $1983 \mathrm{cit}$. Gracia, 1997:65):

- Se manifiestan en el grupo los refuerzos positivos. En este sentido, el grupo aplaude o recompensa las conductas deseables.

- Cuando se comparten las experiencias, pensamientos y sentimien- 
tos cotidianos con otros miembros del grupo.

- Cuando se ofrece feed-back.

- Cuando los miembros del grupo se refuerzan mutuamente acerca de su capacidad para manejar sus problemas.

- Y cuando se justifican los sentimientos o acciones en respuesta a situaciones determinadas.

En el grupo, los miembros se refuerzan mutuamente acerca de su valía personal. Esto se manifiesta en la comprensión y aceptación de todos los miembros del grupo. Cuando un miembro del grupo describe sus acciones o emociones como raras o anormales, los otros miembros tratan de hacer ver que esa conducta es normal.

\subsubsection{Se intercambia información, consejo y educación}

En los grupos se da y se proporciona la información necesaria que ayuda a las personas a mejorar su capacidad de afrontamiento. Los grupos de autoayuda proporcionan esta información bien de forma directa entre los miembros del grupo, bien de forma indirecta indicándose dónde se puede acudir a recibir dicha información.

Una ventaja de estos grupos, respecto a la transmisión de información, es la composición del grupo. Al estar formados por personas en distintas fases del manejo de su problema, los miembros nuevos pueden beneficiarse y obtener información de otros miembros que han superado ya los primeros pasos.

También se puede recibir información invitando al grupo a técnicos o expertos sobre alguna cuestión que interese a los miembros del grupo, de modo que participen en algunas reuniones y aporten la información que el grupo necesite.

\subsubsection{En el grupo se favorece la reestructuración cognitiva.}

Por un lado, el grupo proporciona a sus miembros una razón y una lógica a sus problemas o fuentes de estrés, así como una forma para manejar esos problemas. En este sentido, en el grupo se amplía el rango de percepciones alternativas de los problemas y de las acciones disponibles para manejarlos.

Por otro lado, el grupo apoya el cambio. De esta forma, al considerar los problemas desde una perspectiva distinta, y al adoptar formas nuevas y más efectivas de afrontamiento, la persona comienza a percibirse como un individuo capaz de manejar las circunstancias que le rodean, incrementándose, así, los sentimientos de valía personal. 


\subsubsection{El grupo cumple funciones de socialización}

Aunque, no es su principal objetivo, numerosos grupos de autoayuda consideran la socialización de sus miembros como un aspecto importante. Como nos señala Katz (1993), la función de los grupos de autoayuda para ayudar a sus miembros a superar los sentimientos de aislamiento social es particularmente importante. La integración en un grupo, con las actividades que se llevan a cabo a través de él, puede ayudar a las personas que atraviesan situaciones problema, que les hayan llevado a estar solas, a participar en su comunidad.

\subsubsection{Las acciones se realizan conjuntamente}

La implicación personal en las actividades colectivas del grupo es una de las características más importantes de los grupos de autoayuda. En estos grupos se espera que cada miembro participe, según sus posibilidades, y no sea mero receptor tal y como ocurre en otros grupos.

\subsubsection{El grupo promueve sentimientos de control, autoconfianza y autoestima.}

Las distintas actividades que tienen lugar en los grupos de autoayuda ayudan a superar la pasividad y a mejorar la autoestima, así como a promover la responsabilidad personal.

Tal y como veíamos antes, la adquisición de información, el cambio en las percepciones, la utilización de la propia experiencia para ayudar a los miembros del grupo y las demás características que hemos nombrado ayudan a los miembros del grupo a reforzar la imagen que poseen de ellos mismos.

Como señalan diversos autores (Cameron 1992), los grupos de autoayuda al permitir no sólo la recepción de ayuda sino también su provisión, al promover la participación conjunta de sus miembros en el proceso de ayuda y al incrementar la red natural de apoyo de sus miembros son capaces de incrementar los sentimientos de control de sus miembros sobre su propia vida y de estimular el desarrollo de su autoestima.

\subsection{Tipos de grupos de autoayuda}

El desarrollo de un sistema de clasificación donde se describan los diferentes grupos de autoayuda es tarea imposible. La gran diversidad de grupos de autoayuda, así como la constante proliferación de grupos nuevos, hace que sea imposible poder ofrecer una clasificación. En 
España y más concretamente desde el Trabajo Social, aparecen tantos grupos como diversidad de problemas se traten, como objetivos se planteen o según las diferentes «formas» de entender estos grupos.

La mayoría de grupos que se potencian desde el Trabajo Social comienzan siendo grupos de apoyo donde la dirección corre a cuenta de un profesional para pasar, en un segundo momento y cuando el grupo esté preparado, a funcionar sin la dirección de ningún profesional, esto es, a regirse y funcionar como grupos de autoayuda.

No obstante, en el cuadro $\mathrm{n}^{\circ} 2$ se ofrecen algunas de las clasificaciones que sobre el tema se han propuesto, haciendo hincapié en cuatro de los criterios que consideramos importantes.

CUADRO N. 2

\begin{tabular}{|l|l|l|}
\hline AUTOR & CRITERIO & TIPOS DE GRUPO \\
\hline $\begin{array}{l}\text { LEVY } \\
(1976)\end{array}$ & Composición y objetivos. & $\begin{array}{l}\text { - Grupos para la reorganización de la con- } \\
\text { ducta. } \\
- \text { Grupos de manejo del estrés. } \\
- \text { Grupos orientados a la acción social. } \\
- \text { Grupos de realización personal. }\end{array}$ \\
\hline $\begin{array}{l}\text { UFBRRMAN } \\
(1978)\end{array}$ & Tipo de servicio. & $\begin{array}{l}\text { - Grupos que sirven a sus miembros. } \\
- \text { Grupos que sirven a la sociedad. }\end{array}$ \\
\hline $\begin{array}{l}\text { BARRÓN } \\
(1988)\end{array}$ & $\begin{array}{l}\text { Persona que padece el pro- } \\
\text { blema. }\end{array}$ & $\begin{array}{l}\text { - Los miembros del grupo padecen directa- } \\
\text { mente el problema. }\end{array}$ \\
\hline $\begin{array}{l}\text { LILLALBA miembros del grupo no son afectados } \\
\text { (1996) }\end{array}$ & Destinatarios prioritarios. & $\begin{array}{l}\text { - Aislamiento social. } \\
- \text { Adicciones } \\
- \text { Enfermedad física o mental. } \\
\text { - Supervivientes de catastrofes, atentados... }\end{array}$ \\
\hline
\end{tabular}

Una vez vistas y analizadas las características de los grupos de autoayuda, así como algunas de las clasificaciones que sobre el tema se han propuesto, vamos a exponer cómo puede formarse un grupo de autoayuda desde el Trabajo Social.

Para ello, retomamos la idea que exponíamos antes para recordar que la propuesta que presentamos supone un proceso gradual de creación de un grupo de autoayuda donde el Trabajador social estaría presente en las primeras fases del grupo para acabar fuera del grupo, siendo utilizado en algunas ocasiones a petición expresa del grupo.

Nuestra propuesta, siguiendo el análisis que plantea Barrón (apoyándose en Tylor y col. 1988), defiende que los grupos de apoyo y los de autoayuda pueden considerarse no como dos clases de grupos sino 
como fases en el desarrollo de los grupos basados en el apoyo social. De este modo, habrá grupos que toda su vida estén dirigidos por un profesional, esto es, grupos de apoyo o en fase de apoyo, y habrá grupos que, superado esta fase de apoyo, lleguen a dirigirse de forma autónoma, lo que hemos denominado grupos de autoayuda.

Desde estas premisas, tal y como hemos visto, dada la variedad de grupos y estilos de trabajo profesional, esta propuesta pretende ofrecer una de las posibles líneas de intervención y colaboración con los grupos de autoayuda englobados estos en la perspectiva del apoyo social de la que hablábamos en la primera parte de la exposición. La propuesta que presentamos recoge las ideas que al respecto se han propuesto por Roca y Villalbi (1993) y por Villalba (1996).

\subsection{Metodología del trabajo en grupo}

Centrándonos en la función del profesional en los grupos de autoayuda, estos quedan divididos en cuatro fases o etapas, que se resumen a continuación. Se trata de ver las características de cada fase, así como las funciones del trabajador social para con el grupo.

\section{$I^{a}$ FASE: la creación de un grupo de autoayuda.}

La iniciativa de la puesta en marcha de un grupo de autoayuda puede venir del profesional que detecta la necesidad o problema y ve adecuado una intervención desde el nivel grupal, o puede venir a iniciativa de algunos futuros miembros del grupo.

Tanto si la idea parte del profesional como si no, se debe iniciar un período preparatorio donde todas las personas interesadas en formar parte del grupo tomen contacto unas con otras, comprendan el sentido y el funcionamiento del grupo, así como que se comprometan con y para trabajar en grupo.

Durante estaś reuniones de carácter informal, como el grupo no está todavía formado, no se deben plantear objetivos específicos y actividades, ya que estas deben ser elaboradas por el conjunto de los miembros del grupo una vez ya formado. Lo que sí es importante es que los futuros miembros del grupo comprendan de manera general qué es la autoayuda y qué significa participar en un grupo de autoayuda.

A esta fase preparatoria del trabajo grupal, caracterizada por ser una fase de «captación» de los futuros miembros del grupo, le precede un paso previo dedicado a la difusión y propaganda de la creación del grupo.

Cada vez más, desde distintos ámbitos, se potencia el trabajo en grupos de autoayuda, donde previamente se ha avisado a todas las per- 
sonas que pueden trabajar en este tipo de grupo, preparándoles para el trabajo grupal.

La difusión puede realizarse por diversas vías, en algunas ocasiones se mandan cartas a familiares directamente implicados en distintas situaciones problema para los cuales el grupo de autoayuda puede ser un recurso adecuado. Por ejemplo, cuando desde el hospital se informa a los familiares de personas con problemas de anorexia, de la próxima creación de un grupo de autoayuda, o cuando desde una asociación de enfermos de Sida, se difunde a través de la propia revista de la asociación, la creación de un grupo de autoayuda para voluntarios que prestan su servicios en ella.

\section{$2^{a}$ FASE: primeros contactos con el grupo.}

En esta segunda fase, el objetivo fundamental es ir formando el clima grupal así como que el grupo vaya fijando algunos de los elementos más importantes.

El Trabajador social posee un rol activo, donde debe iniciar progresivamente el proceso de consolidación del grupo.

Durante esta fase, que puede durar 4 sesiones, los miembros del grupo deben conocerse entre sí, asî como conocer de manera más directa qué es la autoayuda y cuál va a ser la metodología de las sesiones, metodología que debe concretar el propio grupo en el transcurso de esta fase.

Los miembros del grupo han de elaborar objetivos, normas, concretar actividades, así como decidir el número de miembros que van a tener, cuáles van a ser las condiciones de ingreso, dónde y cuándo van a reunirse, etc.

Durante el transcurso de esta fase, el Trabajador Social debe dejar progresivamente que el grupo tome la iniciativa, delegando funciones en algunos miembros del grupo.

Entre los objetivos generales de estos grupos tenemos:

1. En primer lugar, conseguir que los miembros del grupo se autorresponsabilicen en sus propios procesos de cambios a través de compromisos cotidianos establecidos por ellos mismos de una sesión a otra.

2. Ayudar, a través de las sesiones, a tomar conciencia de los recursos y déficits individuales relacionados con el tema común del grupo.

3. Incrementar la calidad de apoyo emocional e instrumental a través de una estructura de sesiones que posibilite la expresión, la escucha y la posibilidad de dar y recibir feedback en todos los participantes del grupo. 
4. Y, por último, aumentar las redes sociales de los participantes del grupo a través de la periodicidad de los encuentros y la permanencia de los miembros en el grupo.

No obstante, además de estos objetivos generales, el grupo debe formular objetivos específicos y debe decidir qué tipo de actividades se llevarán a cabo para conseguir los objetivos propuestos.

Teniendo en cuenta que el grupo de autoayuda funciona como tal cuando consigue su propia autonomía, es necesario que el trabajador social desde estas primeras reuniones adopte el criterio de preparar las sesiones con algún miembro del grupo de forma rotativa, de modo que poco a poco sea esta la metodología que se utilice en el grupo y que el trabajador social vaya perdiendo protagonismo.

Este miembro del grupo, al que algunos autores llaman facilitador (Ródenas, 1996:197), posee dos funciones importantes: la de preparar y moderar las sesiones y la de servir de nexo de unión entre el interior y el exterior del grupo.

En algunos grupos de autoayuda esta persona que ejerce el papel de facilitador es siempre la misma, el grupo la elige democráticamente y puede recibir una formación previa por parte de la organización a la que pertenece o a través de cursos de formación para voluntarios. No obstante, consideramos más enriquecedora la experiencia cuando esta tarea es compartida de forma rotativa entre los miembros del grupo, de modo que no cargamos toda la responsabilidad en un sólo miembro del grupo, haciendo mucho más partícipes a estos en su propio grupo.

\section{$3^{a}$ FASE: fase de acompañamiento profesional.}

Una vez establecidos los aspectos formales del grupo así como consolidado el grupo, en esta tercera fase el objetivo fundamental consiste en enseñar al grupo a manejar adecuadamente los aspectos señalados en las sesiones anteriores. De forma más práctica, el grupo debe entrenarse en la práctica de la metodología de autoayuda, enseñando a cada miembro y al grupo en general a comunicarse.

Durante esta fase, que puede durar 12 sesiones, el profesional estará presente en el grupo, intentando no dirigir sino ejercer funciones de acompañamiento y apoyo en el grupo. El profesional preparará las sesiones con el miembro del grupo que le corresponda y será este quien coordine la sesión. Sólo cuando sea necesario, el profesional intervendrá intentando no dar soluciones sino generando la comunicación entre los miembros del grupo, apoyando al moderador en cuanto animar a algún miembro del grupo a hablar etc.

Es interesante la propuesta de Villalba (1996) en cuanto a la forma 
de dar apoyo a los miembros del grupo. Para ella, el profesional debe colocarse detrás de cada miembro del grupo que habla, poniendo o no las manos sobre sus hombros, dependiendo de si percibimos que esta actitud de cercanía física le puede o no ayudar en su comunicación. Esto se hace para apoyar a cada miembro del grupo y demostrar que el profesional que acompaña no es un miembro del grupo, ni un coordinador del mismo sino un asesor y colaborador.

Tanto si se adopta esta disposición física como si no, el profesional puede interrumpir a la persona y orientarle sobre su manera de comunicar al grupo. Por ejemplo, puede decirle que personalice más, que no hable de personas ausentes, que hable de lo que siente, que hable un poco más o bien que dé tiempo para hablar a los otros.

En el transcurso de una reunión, parece conveniente que se den tres momentos importantes:

1. Un primer momento donde cada miembro del grupo expresa al grupo cómo se encuentra, y destaca algún hecho importante que le haya ocurrido desde la anterior sesión. También, cada miembro del grupo comenta si ha realizado el compromiso o la tarea propuesta en la anterior reunión, si es que se ha propuesto alguna.

2. Un segundo momento donde el coordinador explica la preparación de la reunión, así como los temas que propone y los miembros del grupo eligen un tema de los expuestos o bien se puede trabajar acerca de un tema escogido en la anterior reunión donde se ha podido invitar a alguien de fuera.

3. Un tercer momento, donde se realiza una pequeña evaluación de la sesión, así como se proponen tareas o compromisos concretos para realizar hasta la próxima reunión. A la hora de la evaluación el profesional participa aportando ideas sobre el contenido y la metodología que ayuden al grupo en sus próximas sesiones.

$4^{a}$ FASE: fase de observación en el grupo.

En esta cuarta fase, el papel del profesional queda relegado al de observador pasivo del grupo. Es importante que el profesional permanezca en la misma sala del grupo, pero que no se siente con él en círculo, sino que se sitúe fuera del grupo. El Trabajador social actúa como observador, tomando algunas notas sobre cómo se desarrolla la sesión. Es importante que se fije en las tareas de liderazgo, que vea si algún miembro del grupo queda excluido, cómo se llevan a cabo las tareas propuestas, así como que observe de manera especial al miembro que coordina el grupo.

Durante esta fase, que puede durar 3 ó 4 sesiones, según se considere, el objetivo fundamental es que el grupo lleve a cabo la sesión sin la 
presencia cercana ni la participación del profesional. Al igual que en la fase anterior, el profesional, al finalizar la sesión, puede hacer algún comentario sobre lo observado prestando especial atención a la persona que ha coordinado la reunión.

\section{$5^{a} F A S E$ : fase externa o de autodirección.}

En esta quinta fase, que puede durar 3 ó 4 sesiones más, el grupo debe tener ya la suficiente autonomía para poder funcionar por sí solo, como un grupo de autoayuda.

Para ello, el profesional está en otra habitación distinta de donde se lleva a cabo el trabajo grupal, de modo que las reuniones se llevan a cabo sin su presencia.

Para la preparación de las sesiones cada coordinador debe tener en cuenta los aspectos planteados en el grupo, y cada vez menos se pide la ayuda del profesional. No obstante, en estas sesiones previas a la autonomía total, el profesional puede desempeñar el rol de supervisor externo y asesor. Tras cada reunión, el profesional puede reunirse con el coordinador del grupo si lo considera conveniente para ayudarle a realizar una evaluación.

Tras estas 5 fases (resumidas en el cuadro $\mathrm{n}^{\circ} 3$ ), el grupo puede funcionar ya como grupo de autoayuda, teniendo el apoyo del profesional, cuando el grupo lo necesite, ante alguna situación de crisis o conflicto o ante algún cambio importante que ocurra en el grupo.

Este proceso de acompañamiento profesional hacia los grupos permite aprender a trabajar en grupo de forma progresiva y gradual, de ahí que para formar un grupo de autoayuda sea importante que lo tengamos en cuenta. La duración de cada fase no tiene porqué ser fija, ya que depende del tipo de grupo y de las características de sus miembros. No obstante, Villalba propone como duración total del acompañamiento profesional el período comprendido entre seis meses y un año, aunque son fechas aproximadas que dependen, entre otros, de la frecuencia de las reuniones, de las características del grupo y de la participación de los miembros. De todas formas, es importante acordar con los miembros del grupo cuánto tiempo va a durar el acompañamiento profesional. 
CUADRO N. ${ }^{\circ} 3$

1. ${ }^{a}$ FASE: Creación del grupo de autoayuda

0

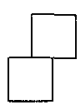

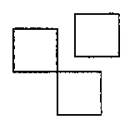

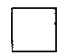

CARACTERÍSTICAS:

- Difusión y captación de los miembros del grupo.

- Información a través de contactos informales.

ROL DEL T.S.:

- Informativo.

2. ${ }^{a}$ FASE: Primeros contactos con el grupo

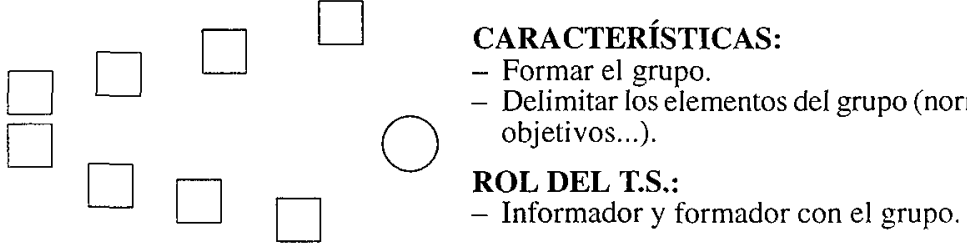

3. ${ }^{a}$ FASE: Fase de acompañamiento personal

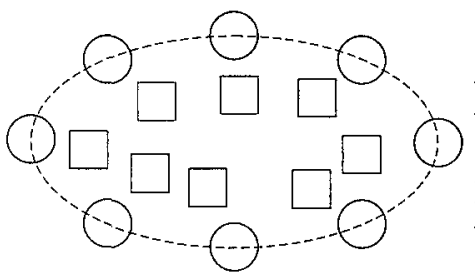

CARACTERÍSTICAS:

- Aprender la metodología de trabajo.

- Entrenar habilidades de comunicación en el grupo.

ROL DEL T.S.:

- Rol de apoyo.

4. ${ }^{a}$ FASE: Fase de observación en el grupo

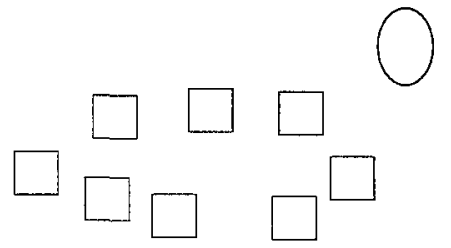

CARACTERÍSTICAS:

- El grupo se autogobierna.

- Independencia de los coordinadores de grupo.

ROL DEL T.S.:

- Observador pasivo.

5. ${ }^{\alpha}$ FASE: Fase externa o de autodirección

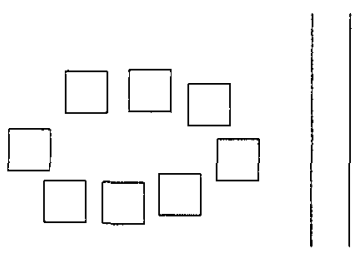

CARACTERÍSTICAS:

- El grupo se autogobierna.

ROL DEL T.S.:

- Supervisor externo y asesor. 


\section{III.CONCLUSIONES}

Los grupos de autoayuda suponen un recurso importante a utilizar desde el Trabajo Social. En ellos se crean vínculos sólidos y se amplían las redes sociales de sus miembros. En este sentido, está comprobada la eficacia de estos grupos para sus miembros. Tal y como señalan Roca y Villalbí (1989: 49), estudios sobre enfermos de cáncer demuestran que los que han asistido a un grupo de autoayuda han sido más capaces de afrontar su situación, asimismo, determinados grupos de autoayuda para ancianos muestran como se mejora la autoestima de sus participantes y la capacidad para realizar actividades.

Estos grupos generan nuevas perspectivas de futuro y capacitan para emprender acciones solidarias. Por ejemplo, muchos de los ex-participantes de un grupo de autoayuda se convierten en voluntarios sociales y siguen apoyando a personas que pasan por la misma situación que ellos pasaron. Esta es una de las características más relevantes de estos grupos. El poder no sólo compartir problemas sino también compartir cómo se pueden enfrentar desde la experiencia supone una fuente de recursos inagotables para los miembros del grupo ofrecidos desde el grupo.

Desde los distintos ámbitos del Trabajo social, el trabajo en grupo, y más concretamente en y con grupos de autoayuda, consolida el trabajo comunitario que desde la práctica se plantea. Estos grupos permiten la participación directa de nuestros clientes en sus problemas facilitando que, a la vez, estos participen en sus propios cambios y ayuden a otros a través del proceso del grupo.

El trabajo a través de grupos de autoayuda en el ámbito de los Servicios Sociales permite una mejora en la calidad de la ayuda, consiguiendo, a su vez, un aumento de la autoestima y una mayor participación en la comunidad de los grupos más desfavorecidos. Desde ellos, y en consecuencia con lo anterior, la dependencia institucional se reduce en el momento que la persona se responsabiliza y se le facilita un marco donde poder tomar decisiones acerca de su situación, situación que es compartida por otras personas.

El papel del profesional en estos grupos, tal y como hemos señalado, ha de ser de asesoramiento, formación y apoyo de líderes y no de liderazgo o coordinación de los mismos. Así pues, debe recordarse que una vez creado y apoyado, el profesional debe mantenerse al margen, porque, tal y como afirma Levin (1977), el beneficio real de los grupos de autoayuda está en su carácter no profesional, en su universalidad, en su accesibilidad, en su diversidad y en su aceptación. 


\section{BIBLIOGRAFÍA}

AYUNTAMIENTO DE MADRID, Área de Servicios Sociales: Trabajo Social con grupos. Teoría y práctica en los Servicios Sociales Municipales. Madrid. 1995.

AZNAR y otros: «Un año de grupo de autoayuda con familiares de enfermos mentales en Zaragoza». Trabajo Social y Salud, 20. Marzo 1995. Pág. 291-296.

BARRÓN, A. (1996): Apoyo social. Aspectos teóricos y aplicaciones. Siglo XXI. Madrid.

BORMAN, L.B. (1992): «Self-help/mutual aid groups in strategies for healt» en KATZ, A. y HEDRICK, H.L.: Self-help: Concepts and applications. The Carles Press. Filadelfia.

CAPLAN, G. y KILLILEA, M. (1976): Support Systems and mutual help. Grune \& Stratton. Nueva York.

ESPALLARGAS, A.: «Nuestra experiencia personal en un grupo de padres». Integración, 16. Octubre, 1994. Págs. 66-67.

FINN, J.: «Computer-Based Self-Help Groups: A new resource to supplement support groups». Social Work with groups, vol.18, 1. 1995.109-116.

GARCÉS TRULLENQUE, E. y otros.: «Grupos de aconsejamiento de padres de pacientes anoréxicos/as : una experiencia desde el Trabajo Social». Trabajo Social y Salud, 28. Diciembre, 1997. Págs. 129-139

GRACIA FUSTER, E. (1997): «Grupos de apoyo y atoayuda, una fuente de recursos para la intervención social» en El apoyo social en la intervención comunitaria. Paidós. Barcelona. Págs. 44-70.

GRACIA, E.; HERRERA, J.; MUSITU, G. (1995): El apoyo social. PPU. Barcelona.

KATZ, A.: «Self-help and mutual aid: An emerging social movement?». Anual Review of Sociology, 7. 1981. Págs. 129-145.

MIRANDA, M. y DE PEDRO, D.: «El concepto de red de apoyo y su importancia para el Trabajo Social». Trabajo Social y Salud, 20. Marzo, 1995.

RIESSMAN, F. (1995): Redefining Self-Help. Police and practice. JosseyBass Publishers. San Francisco.

ROCA, F. y VILLALBI, J.R. (1993): Manual para el funcionamiento de grupos de ayuda mutua. Fondo de Publicaciones del Gobierno de Navarra. Pamplona. Págs. 5-24.

ROCA, R. y VILLALBI, J.R.: «Los grupos de ayuda mutua en el campo de la salud». Revista de Trabajo Social, 114. 1989. Págs. 45-51. 
RÓDENAS PICARDAT, S.: «Grupos de Ayuda Mutua: Una práctica alternativa en la práctica del Trabajo Social».Alternativas, 4. Octubre 1996. Págs. 195-222.

SÁNCHEZ FERNÁNDEZ, C.: «Grupo de apoyo para madres adolescentes». Trabajo Social y Salud, 23. Marzo 1996. Págs. 49-57

SÁNCHEZ MARTÍNEZ, M.L.: «EL trabajo social en las actuaciones grupales con padres y madres ante el fenómeno de las drogodependencias: necesidad de una metodología participativa». Cuadernos de Trabajo Social, 8. Universidad Complutense. Madrid. 1995. Págs. 181-198.

SLUZKI, C. (1996): La red social: Frontera de la práctica sistémica. Gedisa. Barcelona.

TSCHORNE, P. (1995): Dinámica de grupo en Trabajo Social, atención primaria y salud comunitaria. Amarú. Barcelona.

VILLALBA QUESADA, C. (1993): «Redes sociales: un concepto con importantes implicaciones en la intervención comunitaria». Intervención psicosocial, Vol II, 4. Págs. 69-85.

VILLALBA QUESADA, C. (1996): «Los Grupos de Apoyo basados en la autoayuda: una propuesta para el inicio y acompañamiento profesional». Intervención psicosocial. Vol. V, 15. Págs. 23-41. 